\title{
On the integrated PV hosting capacity of MV and LV distribution networks
}

Link to publication record in Manchester Research Explorer

\section{Citation for published version (APA):}

Ballanti, A., \& Ochoa, L. F. (2015). On the integrated PV hosting capacity of MV and LV distribution networks. In IEEE/PES Innovative Smart Grid Technologies ISGT Latin America 2015 (pp. 1-5)

\section{Published in:}

IEEE/PES Innovative Smart Grid Technologies ISGT Latin America 2015

\section{Citing this paper}

Please note that where the full-text provided on Manchester Research Explorer is the Author Accepted Manuscript or Proof version this may differ from the final Published version. If citing, it is advised that you check and use the publisher's definitive version.

\section{General rights}

Copyright and moral rights for the publications made accessible in the Research Explorer are retained by the authors and/or other copyright owners and it is a condition of accessing publications that users recognise and abide by the legal requirements associated with these rights.

\section{Takedown policy}

If you believe that this document breaches copyright please refer to the University of Manchester's Takedown Procedures [http://man.ac.uk/04Y6Bo] or contact uml.scholarlycommunications@manchester.ac.uk providing relevant details, so we can investigate your claim.

\section{OPEN ACCESS}




\section{On the Integrated PV Hosting Capacity of MV and LV Distribution Networks}

\author{
Andrea Ballanti \\ The University of Manchester \\ Manchester, UK \\ andrea.ballanti@postgrad.manchester.ac.uk
}

\author{
Luis F. Ochoa \\ The University of Manchester \\ Manchester, UK \\ luis_ochoa@ieee.org
}

\begin{abstract}
The increasing adoption of residential-scale PV units raises concerns about the potential interactions with MV networks as large volumes of generation connected to one voltage level can limit the hosting capacity of the other. This work investigates such interactions from an integrated MV-LV perspective by analyzing the impacts that different $P V$ penetrations in $L V$ networks have on the hosting capacity of a real UK MV network. Realistic time-series load and PV profiles for a summer day are considered. It was found that the major limitation on the hosting capacity of MV-connected PV units is due to voltage issues of $L V$ customers. In addition, results show that the largest MV-LV aggregated hosting capacity can be achieved by having most of the generation connected to $\mathrm{LV}$ rather than MV, highlighting the benefits of small-scale PV. These findings demonstrate the need to adopt more advanced, integrated MV-LV models in future distribution network studies.
\end{abstract}

Index Terms-- Photovoltaic units, low voltage networks, medium voltage networks, MV hosting capacity

\section{INTRODUCTION}

In the last few years the photovoltaic (PV) installed capacity in several countries around the world has experienced an important increase mainly due to government schemes aimed at reducing greenhouse gas emissions. In particular, the UK in the last 5 years has become one of the few countries worldwide with more than $7.5 \mathrm{GW}$ of PV installed capacity [1] and one of the main PV markets in Europe.

The residential-scale PV units connected to the low voltage (LV) distribution networks $(0.4 \mathrm{kV}$ in the UK) represent only around a third of the national installed capacity [1]. The remaining is given by medium-scale units $(>50 \mathrm{~kW})$ likely to be connected directly to the medium voltage (MV) networks $(6.6,11$ and $33 \mathrm{kV})$. Hence, MV and downstream connected LV networks may simultaneously experience high PV penetration levels. Consequently, it is crucial for Distribution Network Operators (DNOs) to understand how the MV hosting capacity may be affected by high volumes of existing residential-scale PV units (and vice versa) so new installations and the corresponding reinforcements (if needed) can be planned accordingly. In addition, by understanding these interactions it is possible to determine the most suitable policies that will ensure the largest overall penetration of PV units.

The quantification of the PV hosting capacity is a widely investigated topic in the literature considering either planning [2-4] or operational perspectives [5-7]. However, no study undertakes an integrated MV-LV approach to quantify the mutual interactions among networks at different voltage levels. In [2,3] the highest hosting capacity is found by defining the best size and location of distributed generation (DG) units connected to MV networks. However, the effect on LV network was not considered. In [4] the impact assessment of different low carbon technologies in LV networks was quantified but the influence of the MV network was not catered for. In [5] the voltage rise due to rooftop PV units was investigated in a synthetic MV-LV Canadian network. However, only LV-connected PV units were considered. In $[6,7]$ decentralized control strategies were developed to accommodate high volumes of DG without triggering voltages or thermal problems. Nonetheless the benefits of such approaches were limited to the MV network disregarding potential problems that the same generation units may cause in LV.

In this work, the MV-LV interactions are investigated considering the impact that different PV penetration in LV have on the hosting capacity of a real UK MV network. This is achieved by adopting a simplified integrated MV-LV network analysis considering realistic generation and demand profiles. In addition, the overall MV-LV hosting capacity (hereafter called "integrated" hosting capacity) is quantified analyzing its trend for different PV penetration levels.

The rest of the paper is organized as follows. Section II describes the methodology developed for the quantification of the hosting capacity. The adopted case study is introduced in section III while the main results are presented in section IV. The assumptions adopted in this work are discussed in section V. Finally, conclusions are drawn in section VI. 


\section{Accepted Paper}

\section{Methodology}

In this section the adopted definitions of PV penetration level and hosting capacity are introduced. Thereafter, the methodology developed for the quantification of the latter is presented.

\section{A. Definition of PV Penetration Level: $P V_{L V}$ and $P V_{M V}$}

The PV penetration level in $\mathrm{LV}\left(P V_{L V}\right)$ is defined here as the percentage of LV customers of every secondary substation, within the analyzed MV network, with a rooftop $\mathrm{PV}$ unit installation. On the other hand, the PV penetration level in $\mathrm{MV}\left(P V_{M V}\right)$ is defined as the size of the MVconnected PV unit. For simplicity, only one MV site was considered in this work to have a PV installation.

\section{B. Definition of Hosting Capacity: $M V_{H C}$ and $I_{H C}$}

The hosting capacity is here defined as the maximum PV penetration that can be connected without triggering neither transformer thermal problems nor customer overvoltages (hereafter simply called "network issues") considering both MV and LV networks.

In particular, the $\mathrm{MV}$ hosting capacity $\left(M V_{H C}\right)$ is the highest $P V_{M V}$ penetration level that is possible to connect whilst the MV-LV integrated hosting capacity $\left(I_{H C}\right)$ is defined as the highest aggregated MV and LV installed capacity.

The adopted definitions of network issues are described as follows.

\section{1) Thermal Problems of LV or MV Transformers}

In this work a transformer (either MV or LV) experiences a thermal problem when the 10-min three-phase loading level exceeds its nominal capacity. For simplicity, no constraints due to reverse power flows were considered.

\section{2) Customer Overvoltages}

In this work an overvoltage problem arises when a customer voltage (either at LV or MV) exceeds the statutory limits imposed by the UK standard BS EN 50160 [8].

a) LV customer

Due to the significant challenge of explicitly modelling dozens or hundreds of LV networks within a given MV network, here the LV busbars at the secondary substations are used as proxies of LV customer voltages. As such, the LV busbar voltage should be compliant with the UK standard BS EN 50160 [8], i.e., $95 \%$ of the supplied voltages (10-min average rms values) within a week are between 1.10 and 0.90 p.u., and never outside 1.10 and 0.85 p.u. For simplicity, given that PV installations lead to voltage rise issues, only the upper limit of 1.10 p.u. is considered.

\section{b) MV customer}

A MV customer is compliant when $99 \%$ of the supplied voltages (10-min average rms values) within a week is 1.15 and between 1.06 and 0.94 p.u., and never outside 0.85 p.u. For simplicity, given that PV installations lead to voltage rise issues, only the upper limit of $1.06 \mathrm{p} . \mathrm{u}$ is considered.

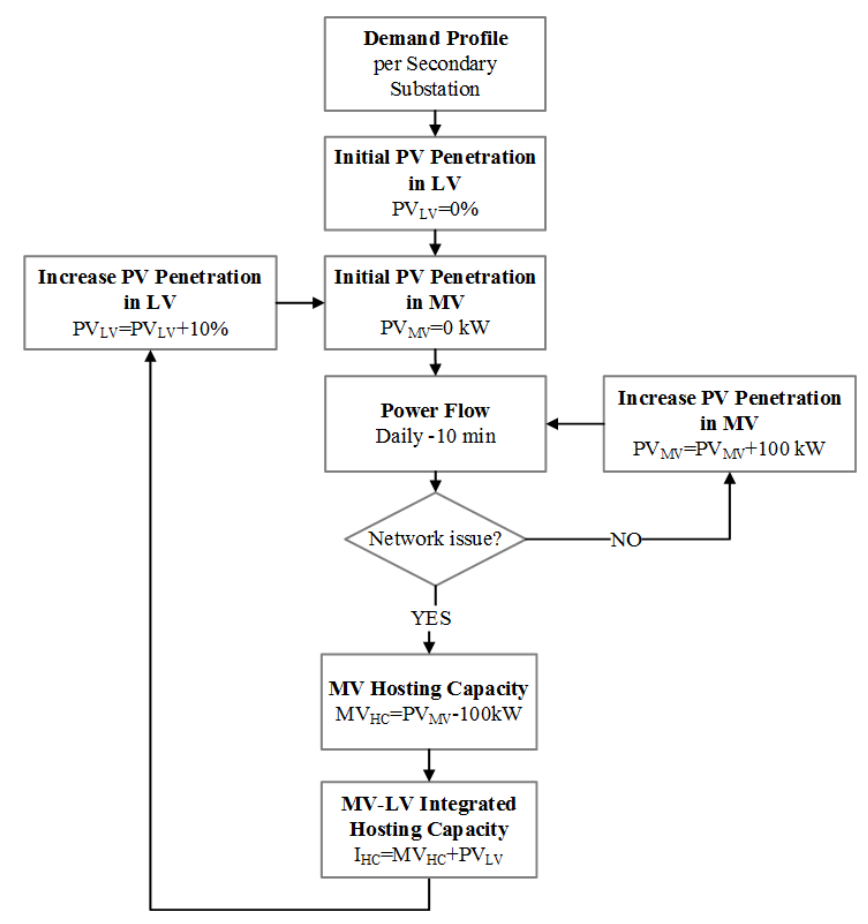

Figure 1 MV-LV integrated hosting capacity quantification: Flowchart

\section{Quantification of the Hosting Capacity}

The methodology adopted to quantify the $\mathrm{MV}\left(M V_{H C}\right)$ and integrated MV-LV hosting capacities $\left(I_{H C}\right)$ is summarized in Figure 1. First, demand profiles per secondary substation within the studied MV network are defined based on, for instance, historical data. Then, the $P V_{L V}$ penetration level (from 0 to $100 \%$ ) and associated generation profiles are fixed. Thereafter, the size of the MV-connected PV unit is gradually increased (from $0 \mathrm{~kW}$ with steps of $100 \mathrm{~kW}$ ) until a network issue is identified by carrying out a 10 -min daily power flow analysis

The MV hosting capacity $M V_{H C}$ corresponds to the $P V_{M V}$ penetration level before the one that triggers network issues. The integrated hosting capacity is then obtained by adding up the MV hosting capacity to the $P V_{L V}$ penetration level, Figure 1. Finally, the whole procedure is repeated for each $P V_{L V}$ penetration level (in $10 \%$ steps) showing the influence that $\mathrm{PV}$ units in LV networks have on the MV one.

The two extreme scenarios in which the PV units are connected exclusively to one voltage level (i.e., with $P V_{L V}$ penetration level equals to 0 and $100 \%$ ) provide extra insights on the interactions among networks. Indeed, with a $P V_{L V}$ penetration of $0 \%$ (MV-only scenario) it is of interest to understand whether the LV networks, even if no PV units are connected at this voltage level, still have an influence on the MV network analysis. Similar studies for the $100 \% P V_{L V}$ penetration level (LV-only scenario) can be performed.

\section{CASE StUdy - Network MOdelling}

This section describes the case study in terms of network models, load and generation profiles that will be used to demonstrate the proposed methodology. 


\section{Accepted Paper}

\section{A. Real UKMV Distribution Network}

A real radial urban MV distribution network $(11 \mathrm{kV})$ from the North West of England is adopted. This network has been modelled into OpenDSS [9] from GIS data. Hence, the real topology, conductor and transformer characteristics were fully considered. In particular, the topology is shown in Figure 2. It consists of 10 feeders that supply around 12,000 customers through 89 secondary substations (single 11/0.433 kV transformers) depicted as green circles in Figure 2. The primary substation (two $33 / 11 \mathrm{kV} 14 \mathrm{MVA}$ parallel transformers) is depicted as a purple square. In addition, the aggregated LV demand and PV generation is considered adopting realistic profiles.

No MV customers are connected in this specific network. Consequently, to cater for the MV customers overvoltage the primary side of every secondary substation is considered as proxy for the MV customers location and, therefore, used to check compliance with the standard BS EN 50160.

\section{B. Load Profiles}

Individual demand profiles for every customer "category" (e.g., residential, commercial, etc.) were adopted in this work. In particular, to realistically reproduce the aggregated residential demand per secondary substation a UK-based tool (CREST tool [10]) is used. Seasonality, number of occupants, geographical location, type of day (i.e., weekend or weekday) and data on energy and power consumption are considered within the tool. The outcome is a minute-by-minute stochastically produced demand profiles per single dwelling. This, in turn, gives realistic variability among the aggregated residential demand per secondary substation.

For the other customer categories (non-residential), halfhourly average demand profiles produced by ELEXON (the UK balancing and settlement company) are used [11].

For every secondary substation the number of supplied customers per category is known. Hence, by combining these numbers with the associated demand profiles previously discussed the aggregated demand per secondary substation was obtained. Figure 3 shows few examples. For consistency with the BS EN 50160 standard the resolution adopted for the profiles is 10 minutes.

\section{PV Generation Profiles}

The generation profiles for PV units are provided by the aforementioned CREST tool. By considering geographical location and cloud coverage it is able to stochastically produce realistic PV profiles for specific days (23 ${ }^{\text {rd }}$ June, weekday).

The residential-scale PV units (all with an installed capacity of $3 \mathrm{~kW}$ ) of LV customers supplied by the same secondary substation are assumed to share the same irradiation profile given the relatively small area (i.e., geographically close to each other). Hence, a total of 89 different irradiance profiles (one per secondary substation in the analyzed MV network) are considered. For the MV-connected PV unit a different generation profile was produced with the CREST tool.

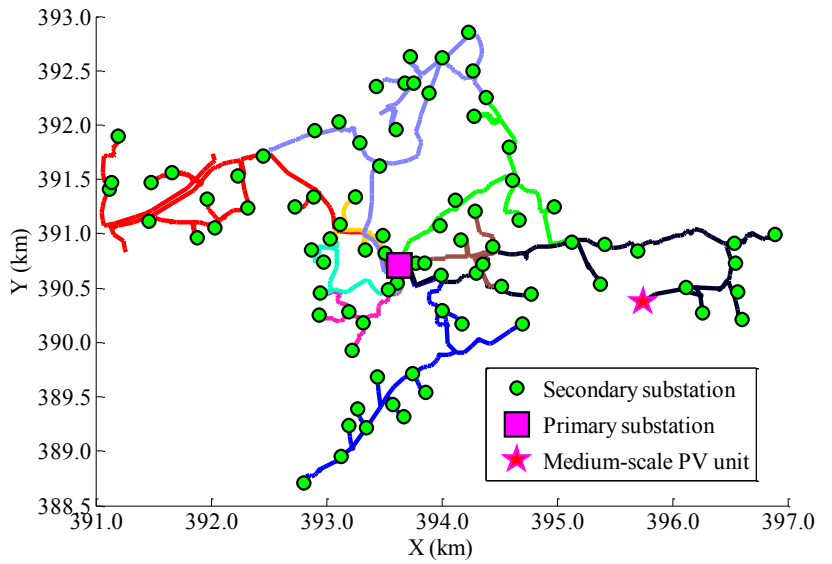

Figure 2 MV network topology (different color per feeder)

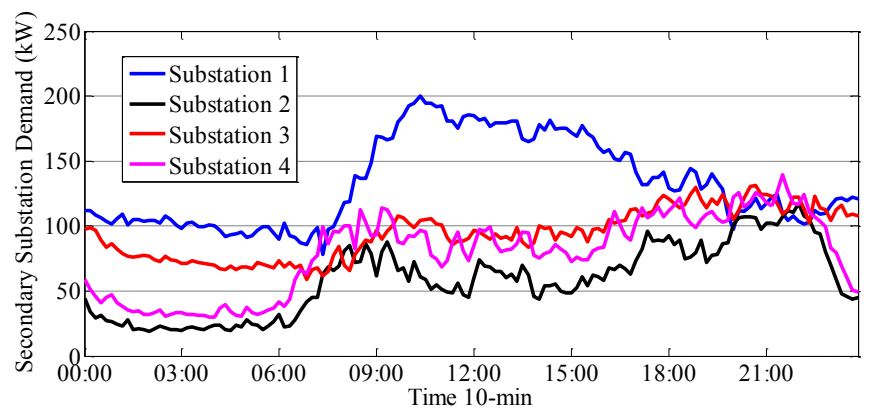

Figure 3 Aggregated demand profile for 4 secondary substations

The MV-connected PV unit is located in one of the furthest points of the MV network (purple star in Figure 2). This allows capturing the highest voltage influence that MV units might have in the surrounding LV networks.

\section{CASE STUDY - RESUlts}

This section firstly reports and discusses the main findings on the hosting capacity $\left(M V_{H C}\right.$ and $\left.I_{H C}\right)$ for the extreme scenarios where PV units are exclusively connected to LV (LV-only) and MV (MV-only) networks. Thereafter, the results for every considered $\mathrm{PV}$ penetration levels $\left(P V_{L V}\right)$ are provided

\section{A. LV-only and MV-only Hosting Capacities}

The integrated hosting capacity $\left(I_{H C}\right)$ given by PV units connected exclusively to LV networks (LV-only) was found to be $20.46 \mathrm{MW}$ (or $60 \%$ if in terms of $P V_{L V}$ ).

In this case, it can be concluded that the main constraint to the hosting capacity in LV is given by voltage problems within the same networks. Indeed, as shown in Table I, no overvoltages appear in MV for any $P V_{L V}$ penetration level. In Figure 4 , the 10-min daily voltage values (minimum, average and maximum) on the primary and secondary side of all LV transformers on the analyzed MV network are summarized. As it can be seen from Figure 4-a the highest LV busbar voltage overcomes the statutory limit (i.e., 1.1 p.u) for PV penetrations above $60 \%$. On the other hand, no voltage problem appears in MV as voltages lay far below the limit of 1.06 p.u, Figure 4-b. 


\section{Accepted Paper}

TABLE I LV-ONLY CASE: $P V_{L V}$ PENETRATION LIMIT PER NeTWORK ISSUE

\begin{tabular}{ccc}
\hline Network Issue & \multicolumn{2}{c}{ Voltage level } \\
& LV & MV \\
\hline \hline $\begin{array}{c}\text { Overvoltage } \\
\text { Thermal }\end{array}$ & $\underline{60 \%(20.46 \mathrm{MW})}$ & none \\
\hline $0 \%(23.87 \mathrm{MW})$ & $90 \%(30.69 \mathrm{MW})$ \\
\hline
\end{tabular}

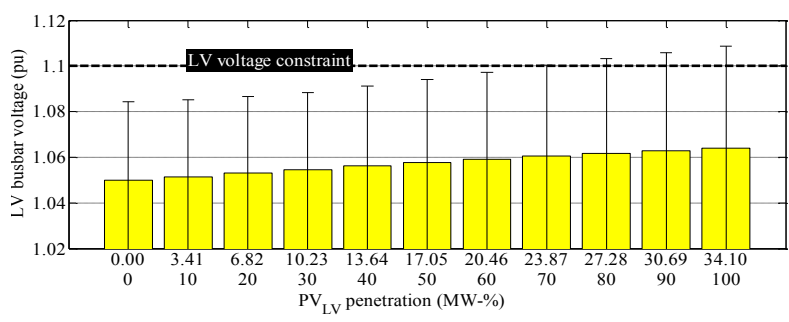

a)

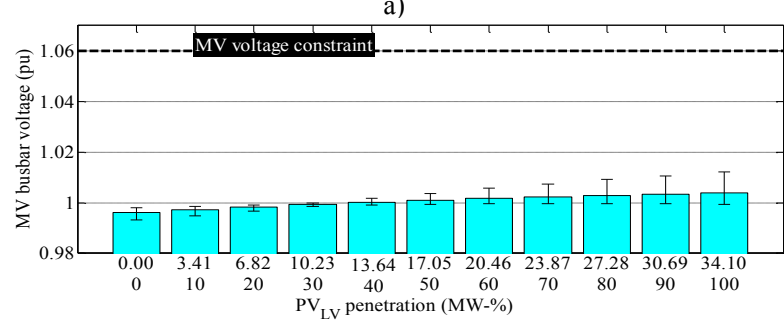

b)

Figure $4 \mathrm{LV}$-only case - LV transformers voltages with $P V_{M V}=0 \mathrm{MW}$ : a) LV busbar b) MV busbar

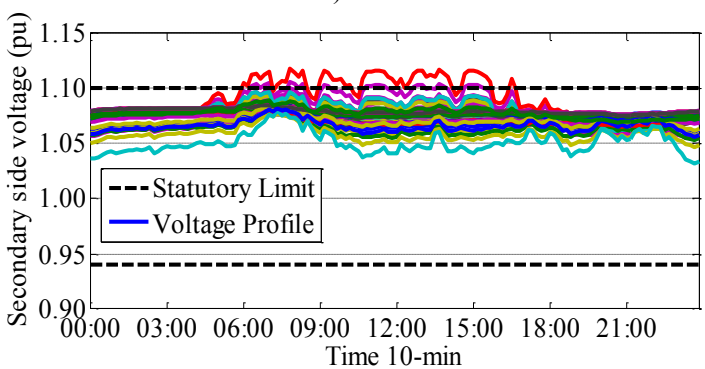

a)

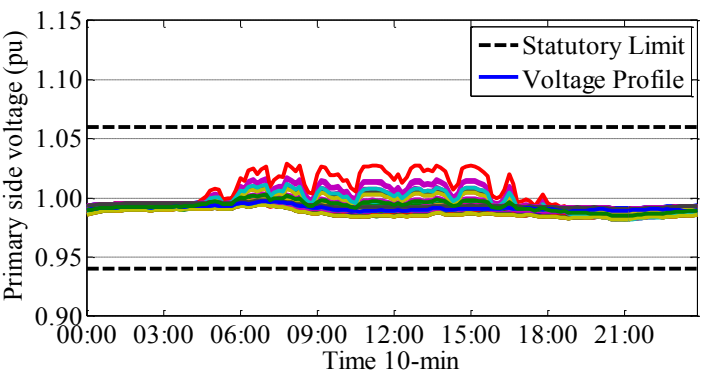

b)

Figure $5 \mathrm{MV}$-only case example- Daily LV transformer voltages with $P V_{M V}=3 \mathrm{MW}$ and $P V_{L V}=0 \%$ : a) LV busbar b) MV busbar

Thermal issues appear in the MV network for penetrations level equal to and greater than $90 \%$ (more than $30 \mathrm{MW}$ ) whilst for a few LV transformers thermal problems were noticed as early as $70 \%$ (more than $23 \mathrm{MW}$ ).

In the MV-only case, the $I_{H C}$ was found to be only 2.4 MW, i.e., around 9 times smaller compared to the LV-only case. It is interesting to remark that the major constraint is again the LV overvoltages even without PV installations in LV networks. Indeed, the MV-connected PV unit quickly

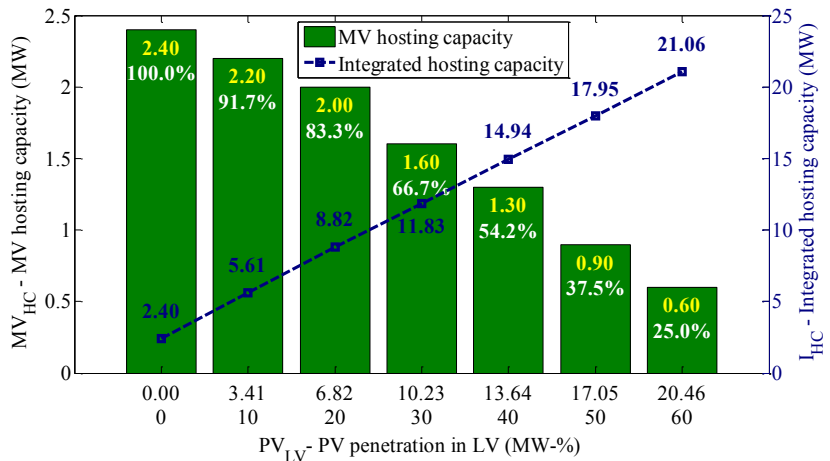

Figure $6 \mathrm{MV}$ and integrated hosting capacity for different $P V_{L V}$

raises the voltage in their surrounding area (due to its large size) leading to voltage problems in LV earlier than in MV.

This effect is clearly shown in Figure 5. In particular, in Figure 5-a, the LV busbar voltages of a few LV transformers (i.e., proxy of the LV customer voltage) were raised above the statutory limit whilst no voltage issues were found in $\mathrm{MV}$ (Figure 5-b). No thermal issues were found in this case.

In the MV-only case it can be concluded that the main constraint to the $I_{H C}$ is given by voltage problems in LV networks. This, in turn, highlights the need in adopting an integrated MV-LV approach even in those cases in which no generation is connected to LV.

In the following section the influence between MV and LV considering PV units simultaneously at both voltage levels is presented and discussed

\section{B. $\quad M V$-LV Integrated Hosting Capacity}

The MV hosting capacity $\left(M V_{H C}\right)$ for different $P V_{L V}$ penetration levels is shown by green bars in Figure 6 as well as with the integrated hosting capacity $\left(I_{H C}\right)$. For instance, with a $P V_{L V}$ penetration level of $20 \%$ (or an aggregated capacity of $6.82 \mathrm{MW}$, third bar in Figure 6) the MV hosting capacity is $2 \mathrm{MW}$ (or $83.3 \%$ of the hosting capacity without $\mathrm{PV}$ in $\mathrm{LV}$ ). The resulting $I_{H C}$ is, therefore, $8.82 \mathrm{MW}$ (blue value in the third bar in Figure 6).

As clearly highlighted by the sharp descending trend in the MV hosting capacity, the PV generation units in LV networks limit significantly the volume of those that can be installed in MV. More specifically, a $10 \%$ increase in the PV penetration in LV leads to approximately an 8 to $16 \%$ reduction of the MV hosting capacity.

These findings demonstrate the need to adopt more advanced, integrated MV-LV models in future network studies. Indeed, limiting the investigation to the same voltage level in which the PV units are connected might lead to misleading results when evaluating the benefits of operational strategies or planning solutions.

Finally, in Figure 6 it can be noticed that the largest MVLV aggregated hosting capacity $\left(I_{H C}\right)$ can be achieved by having most of the PV generation connected to LV rather than MV. In particular, for this case study, it was found that almost 9 times more PV generation can be installed (i.e., from 


\section{Accepted Paper}

2.4 MW to $21.06 \mathrm{MW}$ ) when the PV are almost entirely connected to the LV rather than MV network.

These findings provide a numerical quantification of the well-known benefits of having small PV generation units close to the demand. Consequently, policies that aim at maximizing the overall PV generation should encourage connections of residential-scale PV units in LV rather than medium-scale PV units in MV.

\section{DISCUSSION}

In this section the approximations adopted in this work and the impact on the final results are discussed.

LV network models have not been explicitly included mainly due to their number (89) and lack of data. To overcome this limitation the LV busbar voltage of the secondary substation was adopted as a proxy of customer voltages. However, with high PV penetration levels in LV the voltage of some customers can be even higher than that at the LV busbar. This, in turn, makes the LV networks an even more significant bottleneck to the MV hosting capacity.

In this work a thermal problem was considered to arise when the three-phase power through a transformer exceeds its rating capacity. However, transformers are typically designed to tolerate higher demands for limited periods. Hence, in reality, thermal problems are likely to appear later. On the hand, particularly at primary substations, transformers have reverse power flow limitations. Depending on the overall PV penetration and network characteristics, this could also be a bottleneck.

The primary side of every secondary substation was adopted as a proxy for the MV customer voltages. Consequently, also the nodes characterized by the highest voltages (i.e., close to the MV-connected PV unit) were considered as potential locations. However, in reality, the number of MV customers is typically much lower than the number of secondary substations. This, in turn, makes very unlikely to have MV customers close to the PV unit. Consequently voltage issues in $\mathrm{MV}$ are expected to have an even less influence on the MV hosting capacity.

The numeric results of this work are associated with the specific location of the MV-connected PV unit. In particular, a position closer to the primary substation is expected to results in a higher MV hosting capacity than quantified here (given that voltages at the busbar of primary substations are regulated). Indeed, in such a case, its influence on the surrounding LV networks will be much lower. In addition, the number of MV-connected PV units (only one in this work) will also have an effect on the hosting capacity.

Finally, although the above assumptions might affect the numerical results, they are not expected to influence the key findings of this work.

\section{CONCLUSIONS}

This work investigated the interactions between MV and $\mathrm{LV}$ networks by analyzing the impact that PV units in $\mathrm{LV}$ have on the hosting capacity of a real UK MV network as well as on the integrated MV-LV hosting capacity. Realistic generation and demand profiles have been considered with different PV penetration levels in a 10-min time-series analysis for a summer day.

Results show that LV networks (with or without PV units) represent a major limitation to the hosting capacity that can be connected in MV networks. In particular, it was found that customer voltages in LV are the major bottleneck. Hence, traditional approaches in which the downstream LV networks is neglected might result in overestimations of the actual MV hosting capacity

These findings demonstrate the need to adopt more advanced, integrated MV-LV models in future planning and operational network studies. Indeed, limiting the investigation to the same voltage level in which the PV units are connected might lead to misleading results when evaluating the benefits of potential operational strategies or planning solutions.

It was also found that the largest MV-LV PV installed capacity can be obtained by having most of the generation in $\mathrm{LV}$ rather than in MV. Indeed, small units spread across the LV networks can reach, in the aggregate, much higher volumes compared to a single medium-scale MV-connected PV unit.

Finally, although this work considered only a single MV network, the findings suggest that policies that aim at maximizing the penetration of PV units across a region or a nation should focus efforts on encouraging/incentivizing residential-scale LV connections.

\section{REFERENCES}

[1] DECC, "Solar photovoltaics deployment," 2015

[2] A. Keane and M. O'Malley, "Optimal allocation of embedded generation on distribution networks," IEEE Trans. on Power Systems, vol. 20, pp. 1640-1646, Aug. 2005.

[3] G. Celli, E. Ghiani, S. Mocci, and F. Pilo, "A multiobjective evolutionary algorithm for the sizing and siting of distributed generation," IEEE Trans. on Power Systems, vol. 20, pp. 750-757, May 2005.

[4] A. Navarro-Espinosa and L. F. Ochoa, "Probabilistic Impact Assessment of Low Carbon Technologies in LV Distribution Systems," Power Systems, IEEE Transactions on, vol. PP, pp. 1-12, 2015.

[5] R. Tonkoski, D. Turcotte, and T. H. M. El-Fouly, "Impact of High PV Penetration on Voltage Profiles in Residential Neighborhoods," Sustainable Energy, IEEE Transactions on, vol. 3, pp. 518-527, 2012.

[6] V. Calderaro, G. Conio, V. Galdi, G. Massa, and A. Piccolo, "Optimal Decentralized Voltage Control for Distribution Systems With InverterBased Distributed Generators," Power Systems, IEEE Transactions on, vol. 29, pp. 230-241, 2014.

[7] T. Sansawatt, L. F. Ochoa, and G. P. Harrison, "Smart Decentralized Control of DG for Voltage and Thermal Constraint Management," Power Systems, IEEE Transactions on, vol. 27, pp. 1637-1645, 2012.

[8] BS EN 50160:2010 Voltage characteristics of eletricity supplied by public eletricity networks, B. S. Publication, 2011.

[9] R. C. Dugan and T. E. McDermott, "An open source platform for collaborating on smart grid research," in Power and Energy Society General Meeting, 2011 IEEE, 2011, pp. 1-7.

[10] I. Richardson, M. Thomson, D. Infield, and C. Clifford, "Domestic electricity use: A high-resolution energy demand model," Energy and Buildings, vol. 42, pp. 1878-1887, Oct. 2010.

[11] ELEXON, "Load Profiles and their use in Electricity Settlement," 2015. 